\title{
Local volatility of volatility for the VIX market
}

\section{Journal Article}

Author(s):

Drimus, G.; Farkas, W.

Publication date:

2013-10-01

Permanent link:

https://doi.org/10.3929/ethz-b-000072334

Rights / license:

In Copyright - Non-Commercial Use Permitted

Originally published in:

Review of derivatives research 16(3), https://doi.org/10.1007/s11147-012-9086-9 


\title{
Local volatility of volatility for the VIX market
}

\author{
Gabriel Drimus · Walter Farkas
}

Published online: 8 January 2013

(C) Springer Science+Business Media New York 2013

\begin{abstract}
Following a trend of sustained and accelerated growth, the VIX futures and options market has become a closely followed, active and liquid market. The standard stochastic volatility models - which focus on the modeling of instantaneous variance-are unable to fit the entire term structure of VIX futures as well as the entire VIX options surface. In contrast, we propose to model directly the VIX index, in a mean-reverting local volatility-of-volatility model, which will provide a global fit to the VIX market. We then show how to construct the local volatility-of-volatility surface by adapting the ideas in Carr (Local variance gamma. Bloomberg Quant Research, New York, 2008) and Andreasen and Huge (Risk Mag 76-79, 2011) to a mean-reverting process.
\end{abstract}

Keywords VIX futures · VIX options · Volatility of volatility · Volatility derivatives

JEL Classification $\mathrm{G} 12 \cdot \mathrm{G} 13$

\section{Introduction}

The VIX market - consisting of futures and options on The Chicago Board of Options Exchange (CBOE) VIX index - has been growing at an astounding rate. The average daily volume for VIX options in 2011 has almost doubled compared to 2010 and is nearly 20 times larger than in the year of their launch, 2006. For VIX futures the growth

G. Drimus $(\bowtie) \cdot$ W. Farkas

Institute of Banking and Finance, University of Zürich, Plattenstrasse 14, 8032 Zürich, Switzerland e-mail: gabriel.drimus@bf.uzh.ch

W. Farkas

Department of Mathematics, ETH Zürich, Rämistrasse 101, 8092 Zürich, Switzerland

e-mail: walter.farkas@bf.uzh.ch 

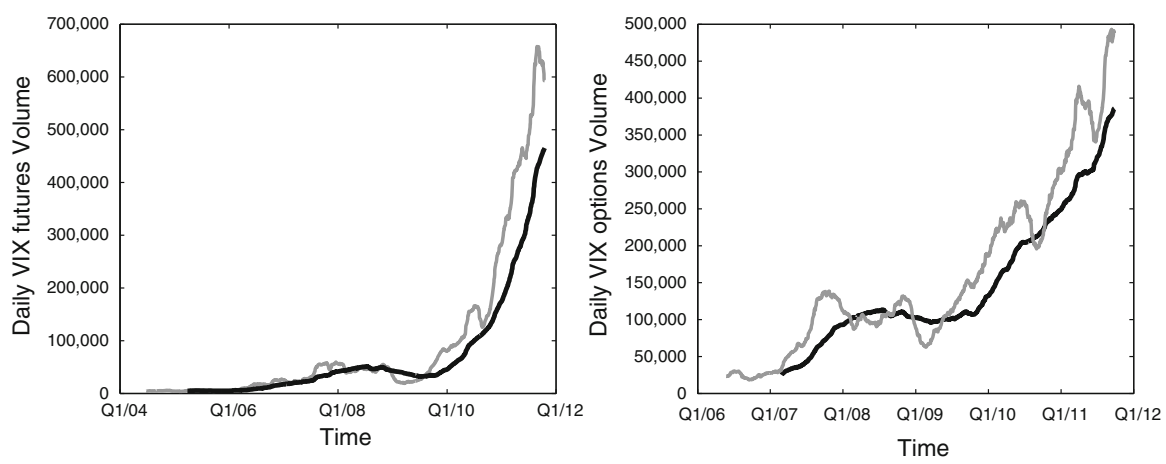

Fig. 1 Daily VIX market volumes: VIX futures contracts ${ }^{1}$ (left), VIX options contracts (right). Black: 252-Day moving average, Gray: 63-Day moving average. Data: $C B O E$

is equally impressive: average daily volume in 2011 has more than tripled compared to 2010 and is about 100 times larger than in the year of their launch, 2004. Figure 1 shows the exponential increase in VIX futures and options volumes since their launch until present: we plot both a 252-day moving average as well as a 63-day (i.e. one quarter) moving average of the daily trading volume. As can be confirmed in Fig. 1, following a sustained and accelerated growth, current average daily volumes amount to almost 1,000,000 contracts/day, ${ }^{1}$ across VIX futures and options.

Similar to the explosive growth in options markets after the seminal work of Black and Scholes (1973), developments in quantitative finance theory played a key role in the birth and growth of volatility markets. Although the CBOE introduced an initial version of the VIX index as early as 1993, it was only after its redefinition in 2003, based on the pioneering work on variance replication of Dupire (1993), Neuberger (1994) and Carr and Madan (1998) that the volatility market started to develop and grow significantly.

Another key driver has been the recognition of volatility, by both traders and investors, as a separate asset class of its own. It was undoubtedly the financial crisis of 2008 that has prominently brought the VIX index to the attention of all market participants; as shown in the study of Szado (2009) volatility was the only asset class which could have offered any diversification benefits, while all other assets were precipitously falling in lockstep.

Recognizing that traditional stochastic volatility models—-such as Scott (1987) and Heston (1993) - lack the required flexibility to simultaneously fit an array of different VIX instruments, the literature has focused on developing more advanced models to jointly fit the S\&P500 and VIX options markets. We particularly remark here the modeling framework introduced in Bergomi (2005) and then further refined in Bergomi (2008) to account for the positive volatility of volatility skew of VIX options. Under a similar framework, Cont and Kokholm (2011) propose dynamics for the spot and forward variances using Levy-processes as building blocks and achieve improved

\footnotetext{
${ }^{1}$ Note: VIX futures contracts have a multiplier of 1000 while VIX options contracts have a multiplier of 100 . Therefore, futures contracts volumes are multiplied by a factor $10 x$, to make them comparable to options contracts volume.
} 
analytical tractability. Such models are particularly useful for exotic index structures, including cliquets and their variations - see also Eberlein and Madan (2009), whose pricing is very sensitive to the volatility-of-volatility. Additionally, these models are suitable for products with payoffs which depend explicitly both on the underlying equity index and its volatility. As expected, however, the capabilities of these models must come at the cost of rather sophisticated dynamics.

In contrast, we deliberately take a more targeted focus and consider only the VIX market in this paper. Specifically, our goal is to find a parsimonious VIX model able to provide a global fit to all listed VIX instruments and which can subsequently be used to price non-listed products, including exotic VIX options such as barrier and digital options. Our main assumption is that the VIX market is mature enough to allow us to price and hedge general VIX derivatives relative to the listed VIX futures and options. In formulating this assumption we rely primarily on the evolution of trading volumes in VIX futures and options on the CBOE market, as discussed in the beginning of this section. Additionally, we consulted with market makers at two leading investment banks who confirmed that at present—unlike a few years ago-the VIX market has improved significantly in terms of both depth and liquidity. Nevertheless, we acknowledge that the evolution of the VIX market is not yet complete and will continue to develop in the coming years; hence, our assumption is likely to find even stronger support over time.

The key distinguishing feature of our approach is that - unlike previous literaturewe will not model either instantaneous variance or forward variances, but rather directly the VIX index itself. Our universe of calibration and hedging instruments will consist of all listed VIX futures and options. For the VIX dynamics, we choose a meanreverting local volatility of volatility model and then show how to obtain a global fit to the VIX market. This new choice of dynamics combines two fundamental ingredients: (1) mean reversion and (2) local volatility. Similar to the classic local volatility literature, without mean reversion—see Derman and Kani (1994), Dupire (1994), Andersen and Brotherton-Ratcliffe (1998) and Andersen and Andreasen (2000)—we obtain the local volatility of volatility function in terms of a surface of VIX options prices. To obtain an arbitrage-free surface, which interpolates and extrapolates the available VIX options quotes, we must properly account for the mean-reversion. To accomplish this task, we show how to adapt the recent ideas in Carr (2008) and Andreasen and Huge (2011) to a process with mean-reversion.

The rest of the paper is organized as follows. In Sect. 2, we specify the meanreverting local volatility-of-volatility dynamics for the VIX index and obtain its key properties. The subsequent section discusses the conditions and the construction of an arbitrage free surface of VIX options prices. In Sect. 4, we present the steps of the numerical calibration of the model to market data. Finally, the last section summarizes the main conclusions. All proofs not given in the main text can be found in the "Appendix".

\section{A new VIX model: mean-reverting local volatility of volatility}

As motivated in the previous section, we will specify directly the dynamics of the VIX index in a mean reverting local volatility of volatility model, thereby combining two 
different ingredients: (1) mean reversion and (2) local volatility. Let $W_{t}$ be a standard Brownian Motion defined on a filtered probability space $\left(\Omega, \mathcal{F}_{t}, \mathcal{F}, \mathbb{Q}\right)$ satisfying the usual conditions. Denoting $\operatorname{VIX}_{t}=V_{t}$, we assume the following dynamics under the risk-neutral measure $\mathbb{Q}$ :

$$
d V_{t}=k \cdot\left(\theta(t)-V_{t}\right) d t+V_{t} \cdot \lambda\left(V_{t}, t\right) d W_{t}
$$

where $k>0$ is the speed of mean-reversion, $\theta(t)>0$ is a deterministic time-dependent reversion level and the local volatility-of-volatility function $\lambda(V, t)$ is assumed sufficiently regular such that the SDE (1) admits a unique non-exploding positive solution.

Our goal is to find $k>0, \theta(t)>0$ and $\lambda(V, t)$ to match the market VIX futures and options. As will become clear in the following, for different fixed values of $k>0$, one can find $\theta(t)$ and $\lambda(V, t)$ to ensure the global fit. Therefore, strictly theoretically, there is flexibility in choosing the parameter $k>0$. In practice, however, a sensible approach could be to select the parameter $k>0$ such that the resulting functions $\theta(t)$ and $\lambda(V, t)$-obtained from the calibration-are reasonably time-homogeneous.

Let us first consider the simpler problem of fitting the VIX futures term structure. We shall take $\theta(t)$ piecewise constant on $0=T_{0}<T_{1}<\cdots<T_{N}$ and denote $\theta(t)=\theta_{i}>0$ on $\left(T_{i-1}, T_{i}\right], i=1,2, \ldots N$, where $T_{1}, T_{2}, \ldots, T_{N}$ are listed maturities for VIX futures and options. An application of Itô's lemma to the process $e^{k t} \cdot V_{t}$ on the interval $[0, T]$ yields:

$$
e^{k T} \cdot V_{T}=V_{0}+k \int_{0}^{T} e^{k t} \theta(t) d t+\int_{0}^{T} e^{k t} V_{t} \lambda\left(V_{t}, t\right) d W_{t}
$$

Assuming the last term is a martingale increment and letting $F_{t}^{T}=E\left(V_{T} \mid \mathcal{F}_{t}\right)$ denote the VIX futures price of maturity $T$, we obtain upon taking expectations:

$$
F_{0}^{T}=e^{-k T} \cdot V_{0}+k e^{-k T} \cdot \int_{0}^{T} e^{k t} \theta(t) d t
$$

For a piecewise constant $\theta(t)$ and for $T \in\left(T_{n-1}, T_{n}\right]$, with $n=1,2, \ldots, N$, we have

$$
\begin{aligned}
F_{0}^{T} & =e^{-k T} \cdot V_{0}+\sum_{i=1}^{n-1} \theta_{i} \cdot e^{-k T} \cdot \int_{T_{i-1}}^{T_{i}} k e^{k t} d t+\theta_{n} \cdot e^{-k T} \cdot \int_{T_{n-1}}^{T} k e^{k t} d t \\
& =e^{-k T} \cdot V_{0}+\sum_{i=1}^{n-1} \theta_{i} \cdot\left(e^{-k\left(T-T_{i}\right)}-e^{-k\left(T-T_{i-1}\right)}\right)+\theta_{n} \cdot\left(1-e^{-k\left(T-T_{n-1}\right)}\right) .
\end{aligned}
$$

In particular, if the maturity $T$ is one of the listed maturities $T_{n}$, the model VIX futures price will be: 


$$
F_{0}^{T_{n}}=e^{-k T_{n}} \cdot\left(V_{0}+\sum_{i=1}^{n} \theta_{i} \cdot\left(e^{k T_{i}}-e^{k T_{i-1}}\right)\right)
$$

Now, let us denote by $F_{0}^{T_{n}, M}$ the VIX futures of maturity $T_{n}$, as observed in the market at time $t=0$. From the condition $F_{0}^{T_{n}}=F_{0}^{T_{n}, M}$ and Eq. (2), we finally obtain $\theta_{n}$ as

$$
\theta_{n}=\frac{F_{0}^{T_{n}, M} \cdot e^{k T_{n}}-V_{0}-\sum_{i=1}^{n-1} \theta_{i} \cdot\left(e^{k T_{i}}-e^{k T_{i-1}}\right)}{e^{k T_{n}}-e^{k T_{n-1}}} .
$$

Applying (3) in turn, for $n=1,2, \ldots, N$, we recover the function $\theta(t)$ which ensures the fit to the VIX futures term structure.

In what follows, we denote by $C(K, T)$ the value of a call option on the VIX index, with strike $K$ and maturity $T$. Specifically, we define

$$
C(K, T) \triangleq e^{-r T} E\left(V_{T}-K\right)_{+}
$$

where $r$ denotes the risk-free interest rate. In analogy to the classic local volatility literature, we want to obtain a relation between the local volatility of volatility function $\lambda(K, T)$ and the call options surface $C(K, T)$ - as it applies to options on a meanreverting underlying, in our case, the VIX index. We arrive at the relation stated in the proposition below.

Proposition 2.1 Under the VIX dynamics (1) and assuming suitable regularity conditions, the local volatility-of-volatility function $\lambda(K, T)$ satisfies:

$$
\lambda^{2}(K, T)=\frac{\frac{\partial C}{\partial T}(K, T)+k(\theta(T)-K) \cdot \frac{\partial C}{\partial K}(K, T)+(r+k) \cdot C(K, T)}{\frac{1}{2} \cdot K^{2} \cdot \frac{\partial^{2} C}{\partial K^{2}}(K, T)} .
$$

Proof The main steps of the proof are similar to the non-mean-reverting case (e.g. Dupire (1994) or Andersen and Brotherton-Ratcliffe 1998). We only remark that the linear form of the mean-reversion drift in (1) is explicitly required in order to arrive at a formula as in (4); for completeness, we include the details in "Appendix I".

As remarked in the proof above, a linear mean-reversion is needed in order to obtain the formula in (4). However, it is interesting to note that volatility dynamics with nonlinear mean-reversion have also appeared in the literature, for example the quadratic mean-reversion of the $3 / 2$ model in Lewis (2000) and Carr and Sun (2007). The results here, including the construction of $\lambda(K, T)$ discussed in the following section, directly depend on the choice of a linear mean-reversion.

In practice, we may find it convenient to work with log-strikes, as opposed to absolute strikes. If we let $x=\log \left(\frac{K}{V_{0}}\right)$ denote the log-moneyness, we can define $\tilde{\lambda}(x, T)=\lambda\left(V_{0} e^{x}, T\right)$ and similarly $\widetilde{C}(x, T)=C\left(V_{0} e^{x}, T\right)$; Eq. (4) can then be rewritten as: 


$$
\widetilde{\lambda}^{2}(x, T)=\frac{\frac{\partial \widetilde{C}}{\partial T}(x, T)+k\left(\theta(T)-V_{0} e^{x}\right) \cdot \frac{1}{V_{0} e^{x}} \cdot \frac{\partial \widetilde{C}}{\partial x}(x, T)+(r+k) \cdot \widetilde{C}(x, T)}{\frac{1}{2} \cdot\left(\frac{\partial^{2} \widetilde{C}}{\partial x^{2}}(x, T)-\frac{\partial \widetilde{C}}{\partial x}(x, T)\right)} .
$$

For the numerical implementation, it is important to have a representation for $\lambda^{2}(K, T)$ (or equivalently for $\widetilde{\lambda}^{2}(x, T)$ ), similar to Proposition 2.1, but in terms of Black-Scholes implied volatilities. However, due to mean-reversion, it is not possible to directly define a Black-Scholes implied volatility for the spot VIX. To see this, we recall the no-arbitrage bounds that must be satisfied by a call option on a non-dividend paying underlying with initial value $V_{0}$ :

$$
\left(V_{0}-e^{-r T} K\right)_{+}<C(K, T)<V_{0}
$$

However, the bounds satisfied by call options on the VIX are:

$$
e^{-r T}\left(F_{0}^{T}-K\right)_{+}<C(K, T)<e^{-r T} F_{0}^{T}
$$

where, as defined previously, $F_{0}^{T}=E\left(V_{T}\right)$ is the VIX futures of maturity $T$. We can now easily see that for sufficiently low strikes $K$ and a futures prices $F_{0}^{T}$ sufficiently larger than the spot VIX value $V_{0}$, the lower bound in (7) would be larger than the upper bound in (6) and hence the Black-Scholes implied volatility would not be well defined. Instead, we shall define the Black implied volatility $\sigma(K, T)$ of the VIX futures $F_{0}^{T}$ :

$$
C(K, T) \triangleq C^{B}\left(F_{0}^{T}, K, T, \sigma(K, T)\right)
$$

where $C^{B}(F, K, T, \sigma)$ denotes the standard Black formula:

$$
C^{B}(F, K, T, \sigma)=e^{-r T}\left[F N\left(d_{1}\right)-K N\left(d_{2}\right)\right]
$$

with

$$
\begin{aligned}
& d_{1}=\frac{\log \left(\frac{F}{K}\right)+\frac{\sigma^{2} T}{2}}{\sigma \sqrt{T}} \\
& d_{2}=d_{1}-\sigma \sqrt{T} .
\end{aligned}
$$

Proposition 2.2 states the formula for the local volatility-of-volatility $\tilde{\lambda}(x, T)$ in terms of Black implied volatilities. We note that this formula bears certain differences from the classical formula in Andersen and Brotherton-Ratcliffe (1998) since, in our case, the futures $F_{0}^{T}$ further depends on the mean-reversion related terms $k$ and $\theta(t)$. For the statement below we let $\widetilde{\sigma}(x, T)=\sigma\left(V_{0} e^{x}, T\right)$, but we drop the tilde sign for brevity. 
Proposition 2.2 Under the assumptions of Proposition 2.1, the local volatility of volatility $\tilde{\lambda}(x, T)$ satisfies:

$$
\begin{gathered}
\tilde{\lambda}^{2}(x, T)=\frac{k \theta_{i}\left(N\left(d_{1}\right)-N\left(d_{2}\right)\right)+F_{0}^{T} n\left(d_{1}\right)\left[\frac{\sigma(x, T)}{2 \sqrt{T}}+\sqrt{T} \cdot \frac{\partial}{\partial T} \sigma(x, T)+\right.}{\frac{F_{0}^{T}}{2} n\left(d_{1}\right)\left[\frac{1}{\sigma(x, T) \sqrt{T}}+\left(\frac{2 d_{1}}{\sigma(x, T)}-\sqrt{T}\right) \cdot \frac{\partial}{\partial x} \sigma(x, T)+\right.} \cdots \\
+\frac{\left.+\frac{k\left(\theta_{i}-V_{0} e^{x}\right) \sqrt{T}}{V_{0} e^{x}} \cdot \frac{\partial}{\partial x} \sigma(x, T)\right]}{\left.+\frac{\sqrt{T} d_{1} d_{2}}{\sigma(x, T)} \cdot\left(\frac{\partial}{\partial x} \sigma(x, T)\right)^{2}+\sqrt{T} \cdot \frac{\partial^{2}}{\partial x^{2}} \sigma(x, T)\right]}
\end{gathered}
$$

where $\theta_{i}=\theta(T)$ for $T \in\left(T_{i-1}, T_{i}\right]$ and $N(\cdot), n(\cdot)$ denote the standard normal cumulative and probability density functions, respectively.

Proof See "Appendix I".

Despite the rather complicated appearance of Eq. (9), it proves useful in the numerical implementation - to be discussed further in the following two sections-as it is often numerically more stable to differentiate the surface of implied volatilities than to differentiate the surface of absolute call prices, as in the simpler formulas (4) or (5).

\section{Constructing the local volatility-of-volatility surface}

Applying Proposition 2.1 (or Proposition 2.2) to compute the local volatility of volatility $\lambda(K, T)^{2}$ requires a full surface of VIX option prices. Consider a finite and discrete strike $\times$ maturity grid on which, for each of the listed maturities $T_{i}$, we have some set of listed strikes $\mathcal{K}(i)$, where there exist VIX options market quotes. Our goal is to construct a VIX options surface which interpolates and extrapolates the available market quotes to the entire grid.

Most importantly, this interpolation and extrapolation procedure must ensure that the resulting VIX options surface is arbitrage free. Consequently, before proceeding to the construction of the options surface, we begin by identifying the relevant noarbitrage conditions. Similar to the non mean-reverting case, we identify appropriate analogues to the calendar spread and butterfly spread conditions, as they apply to VIX options.

The rest of this section is organized as follows. The first subsection summarizes the no-arbitrage conditions for VIX options as formulated in Lemma (3.1). In a subsequent subsection we present a construction algorithm for the VIX options surface by extending ideas in Carr (2008) and Andreasen and Huge (2011) to a mean-reverting process; this task is further broken down in two possible approaches: one which works

\footnotetext{
2 We note that the function $\lambda(\cdot, \cdot)$ is the same function as in Eq. (1) of the previous section, only the notation of the arguments may differ depending on the context.
} 
directly with the spot VIX and one which works indirectly with VIX futures. The section concludes with some remarks regarding the choice between the two construction approaches.

\subsection{No Arbitrage Conditions for VIX options}

Lemma 3.1 VIX options satisfy the no-arbitrage conditions:

1. Calendar spread: for any $T_{1}<T_{2}$

$$
C\left(K, T_{2}\right)-e^{-(r+k)\left(T_{2}-T_{1}\right)} \cdot C\left(K e^{k\left(T_{2}-T_{1}\right)}-e^{-k T_{1}} \int_{T_{1}}^{T_{2}} k e^{k t} \theta(t) d t, T_{1}\right) \geq 0
$$

2. Butterfly spread: for any $K-\Delta K<K<K+\Delta K$

$$
C(K-\Delta K, T)-2 \cdot C(K, T)+C(K+\Delta K, T)>0
$$

Proof See "Appendix I".

In particular, we note that the calendar spread condition takes on a more complicated form due to mean-reversion, whereas the butterfly condition keeps its classical form. The necessary no-arbitrage conditions of Lemma 3.1 can also be shown to be sufficient. To see this, note that dividing (10) by $T_{2}-T_{1}$ then letting $\Delta T=T_{2}-T_{1} \rightarrow 0$ and, similarly, dividing (11) by $\Delta K$ then letting $\Delta K \rightarrow 0$, we obtain:

$$
\begin{aligned}
\frac{\partial C}{\partial T}(K, T)+k(\theta(T)-K) \cdot \frac{\partial C}{\partial K}(K, T)+(r+k) \cdot C(K, T) & \geq 0 \\
\frac{\partial^{2} C}{\partial K^{2}}(K, T) & >0
\end{aligned}
$$

where to ensure the strict inequality in (13), we make the assumption that the density of $V_{T}$ does not vanish on $\mathbb{R}_{+}$. By Proposition 2.1, conditions (12), (13) ensure that the local volatility-of-volatility function is well-defined and, in turn, this implies (up to suitable regularity conditions) that we have well-defined dynamics in (1) consistent with the VIX option prices.

\subsection{Construction based on Carr-Andreasen-Huge with mean reversion}

In constructing the arbitrage-free VIX options surface we start from the following key result based on Carr (2008). 
Theorem 3.1 (Carr (2008)) For a function $F: \mathbb{R} \times\left[0, T^{*}\right] \longrightarrow \mathbb{R}$ which satisfies, for any fixed $T \in\left(0, T^{*}\right]$, the ordinary differential equation $(O D E)$ :

$$
\frac{F(K, T)-F(K, 0)}{T}=\frac{1}{2} \cdot K^{2} \cdot v^{2}(K) \cdot \frac{\partial^{2} F}{\partial K^{2}}(K, T)
$$

where $F(K, 0)$ is convex in $K$, we have:

$$
\frac{\partial F}{\partial T}(K, T)>0 \text { and } \frac{\partial^{2} F}{\partial K^{2}}(K, T)>0
$$

where $v(K)$ is an arbitrary non-vanishing function.

Proof See Carr (2008). For an alternative derivation, we refer to Andreasen and Huge (2011); the latter also includes a discrete version of the result.

Andreasen and Huge (2011) use the result in Theorem 3.1 to develop a fast algorithm to interpolate/extrapolate a finite set of European option prices to a full arbitrage-free surface.

The key feature of Theorem 3.1, is that it prescribes a sequence of ordinary differential equations for $F(K, T)$, as opposed to a partial differential equation; note that it contains no partial derivative in $T$, only a simple finite difference ratio. It resembles closely the classical Dupire (1994) forward PDE, with the crucial difference that the derivative in $T$ is here automatically discretized. This important property is efficiently exploited in Andreasen and Huge (2011) to obtain a fast and robust algorithm for the arbitrage-free construction of an options surface.

For our purposes, we rewrite Eq. (14) as follows. For a function $F: \mathbb{R} \times$ $\left[T_{i-1}, T_{i}\right] \longrightarrow \mathbb{R}$ which satisfies, for any $T \in\left(T_{i-1}, T_{i}\right]$, the ordinary differential equation (ODE) :

$$
\left(1-\frac{T-T_{i-1}}{2} \cdot K^{2} \cdot v_{i}^{2}(K) \cdot \frac{\partial^{2}}{\partial K^{2}}\right) F(K, T)=F\left(K, T_{i-1}\right)
$$

and for which $F\left(K, T_{i-1}\right)$ is convex in $K$, we have:

$$
\frac{\partial F}{\partial T}(K, T)>0 \text { and } \frac{\partial^{2} F}{\partial K^{2}}(K, T)>0
$$

where $v_{i}(K)$ is an arbitrary non-vanishing function.

For further reference, we now briefly recall the key ideas in the volatility interpolation algorithm of Andreasen and Huge (2011). Note that the conditions in (16) are precisely the no-arbitrage conditions which must be satisfied by a strike $\times$ maturity surface of call options-denoted here $F(K, T)$ - on a non-dividend paying, tradeable underlying, under zero interest rates. Start by setting the initial condition: $F(K, 0)=\left(S_{0}-K\right)_{+}$. For each interval $\left[T_{i-1}, T_{i}\right]$, perform steps: 
1. Long step: Search for $v_{i}(\cdot)$ so as to 'hit' the discrete market quotes $F^{M}\left(K_{j_{k}}, T_{i}\right)$ of maturity $T_{i}$ :

$$
\min _{\nu_{i}(\cdot)} \sum_{K_{j_{k}} \in \mathcal{K}(i)}\left(F\left(K_{j_{k}}, T_{i}\right)-F^{M}\left(K_{j_{k}}, T_{i}\right)\right)^{2}
$$

by applying ODE (15) with $T=T_{i}$.

2. Short steps: Fill-in all grid maturities $T \in\left(T_{i-1}, T_{i}\right]$ by using the $v_{i}(\cdot)$ determined in step 1 into ODE (15).

It is important to emphasize the core idea: long-step between listed maturities $T_{i-1}$ and $T_{i}$ in order to find $v_{i}(\cdot)$ and then short-step from $T_{i-1}$ to each $T \in\left(T_{i-1}, T_{i}\right]$ to fill-in the remaining maturity layers. The final result is an options surface which satisfies the conditions (16) and is consistent with the observed market quotes.

We now proceed to develop two methods for constructing an arbitrage-free surface of VIX option prices. Both methods start from the key ideas in Carr (2008) and Andreasen and Huge (2011) by showing two different approaches to accommodate mean-reverting dynamics. The first method works directly with the VIX spot, whereas the second method works with the VIX futures. In both cases, the end aim is to compute the local volatility-of-volatility function $\lambda(K, T)$, consistent with the observed VIX option market quotes.

\subsubsection{Method I: Direct method using spot VIX}

Recall that the VIX options surface must satisfy the no arbitrage conditions (12) and (13). For each interval between listed maturities $\left[T_{i-1}, T_{i}\right]$, we would like to obtain an ODE representation for $C(K, T)$ similar to (15) and which automatically ensures that conditions (12) and (13) are satisfied. To this end, we start from a function $F: \mathbb{R} \times\left[T_{i-1}, T_{i}\right] \longrightarrow \mathbb{R}$ as in (15), and define

$$
C(K, T) \triangleq e^{-(r+k)\left(T-T_{i}\right)} \cdot F\left(\theta_{i}+\left(K-\theta_{i}\right) e^{k\left(T-T_{i}\right)}, T\right)
$$

Setting $T=T_{i-1}$ and taking $K$ as $\theta_{i}+\left(K-\theta_{i}\right) e^{k\left(T_{i}-T_{i-1}\right)}$, we obtain the initial condition at time $T_{i-1}$ as

$$
F\left(K, T_{i-1}\right)=e^{-(r+k)\left(T_{i}-T_{i-1}\right)} \cdot C\left(\theta_{i}+\left(K-\theta_{i}\right) e^{k\left(T_{i}-T_{i-1}\right)}, T_{i-1}\right) .
$$

Differentiating (17) with respect to $K$ and $T$, we also obtain:

$$
\begin{aligned}
\frac{\partial C}{\partial K}(K, T) & =e^{-r\left(T-T_{i}\right)} \cdot \frac{\partial F}{\partial K}\left(\theta_{i}+\left(K-\theta_{i}\right) e^{k\left(T-T_{i}\right)}, T\right) \\
\frac{\partial^{2} C}{\partial K^{2}}(K, T) & =e^{-(r-k)\left(T-T_{i}\right)} \cdot \frac{\partial^{2} F}{\partial K^{2}}\left(\theta_{i}+\left(K-\theta_{i}\right) e^{k\left(T-T_{i}\right)}, T\right)>0 \\
\frac{\partial C}{\partial T}(K, T) & =e^{-(r+k)\left(T-T_{i}\right)} \cdot\left[-(r+k) \cdot F\left(\theta_{i}+\left(K-\theta_{i}\right) e^{k\left(T-T_{i}\right)}, T\right)\right.
\end{aligned}
$$




$$
\begin{aligned}
& +\frac{\partial F}{\partial T}\left(\theta_{i}+\left(K-\theta_{i}\right) e^{k\left(T-T_{i}\right)}, T\right) \\
& \left.+k\left(K-\theta_{i}\right) e^{k\left(T-T_{i}\right)} \frac{\partial F}{\partial K}\left(\theta_{i}+\left(K-\theta_{i}\right) e^{k\left(T-T_{i}\right)}, T\right)\right]
\end{aligned}
$$

Using (18) and (20), we can conclude:

$$
\begin{aligned}
\frac{\partial C}{\partial T}(K, T)+ & k\left(\theta_{i}-K\right) \cdot \frac{\partial C}{\partial K}(K, T)+(r+k) \cdot C(K, T) \\
& =e^{-(r+k)\left(T-T_{i}\right)} \cdot \frac{\partial F}{\partial T}\left(\theta_{i}+\left(K-\theta_{i}\right) e^{k\left(T-T_{i}\right)}, T\right)>0 .
\end{aligned}
$$

Finally, evaluating (15) at $K$ of the form $\theta_{i}+\left(K-\theta_{i}\right) e^{k\left(T-T_{i}\right)}$ and then making use of relation (19), we have proved the following result.

Proposition 3.1 For a function $C: \mathbb{R} \times\left[T_{i-1}, T_{i}\right] \longrightarrow \mathbb{R}$ which satisfies, for any fixed $T \in\left(T_{i-1}, T_{i}\right]$, the ordinary differential equation $(O D E)$ :

$$
\begin{aligned}
& {\left[1-\frac{T-T_{i-1}}{2} \cdot\left(\theta_{i}+\left(K-\theta_{i}\right) e^{k\left(T-T_{i}\right)}\right)^{2} \cdot v_{i}^{2}\left(\theta_{i}+\left(K-\theta_{i}\right) e^{k\left(T-T_{i}\right)}\right)\right.} \\
& \left.\cdot e^{-2 k\left(T-T_{i}\right)} \cdot \frac{\partial^{2}}{\partial K^{2}}\right] C(K, T)=e^{-(r+k)\left(T-T_{i-1}\right)} \cdot C\left(\theta_{i}+\left(K-\theta_{i}\right) e^{k\left(T-T_{i-1}\right)}, T_{i-1}\right)
\end{aligned}
$$

where $C\left(K, T_{i-1}\right)$ is convex in $K$ and $v_{i}(K)$ is an arbitrary non-vanishing function, the following properties hold:

$$
\begin{aligned}
\frac{\partial C}{\partial T}(K, T)+k\left(\theta_{i}-K\right) \cdot \frac{\partial C}{\partial K}(K, T)+(r+k) \cdot C(K, T) & >0 \\
\frac{\partial^{2} C}{\partial K^{2}}(K, T) & >0 .
\end{aligned}
$$

Equation (21) provides an analogue to (15) for the case when the underlying is meanreverting. Using this result, we can now obtain an options surface which satisfies the no-arbitrage conditions (12) and (13), which are the relevant no-arbitrage conditions for VIX options.

We can now apply the same logic of the Andreasen and Huge (2011) algorithm to Proposition 3.1, with an additional adjustment however. To see this, let us assume that we have computed the options surface on $\left[0, T_{i-1}\right]$ for a discrete strike grid $\left\{K_{j}\right\}$ and, hence, have also solved for $\nu_{l}(\cdot)$ for all $1 \leq l \leq i-1$. In particular, we have the values of the call prices $C\left(K_{j}, T_{i-1}\right)$ on our discrete strike grid $\left\{K_{j}\right\}$.

However, note that in order to step from $T_{i-1}$ to any $T \in\left(T_{i-1}, T_{i}\right]$ the initial condition in Eq. (21) will require the call prices $C\left(\cdot, T_{i-1}\right)$ at the discrete strikes $\theta_{i}+\left(K_{j}-\theta_{i}\right) e^{k\left(T-T_{i-1}\right)}$ (but we only have them at the strikes $\left.\left\{K_{j}\right\}\right)$. Since we have already computed $v_{l}(\cdot)$ for all $1 \leq l \leq i-1$, we can obtain these call prices by long-stepping again from time $T_{0}=0$ forwards to $T_{i-1}$, with carefully chosen initial 
conditions. Specifically, we must amend both steps 1 and 2 of the Andreasen and Huge (2011) algorithm, as follows:

- (Initial condition) To obtain call prices $C\left(\theta_{i}+\left(K_{j}-\theta_{i}\right) e^{k\left(T-T_{i-1}\right)}, T_{i-1}\right)$ longstep between $T_{0}, T_{1}, T_{2}, \ldots, T_{i-1}$ using Eq. (21) and the initial condition at time $T_{0}=0, C\left(K_{j}^{\prime}, 0\right)=\left(V_{0}-K_{j}^{\prime}\right)_{+}$where

$$
K_{j}^{\prime}=\theta_{1}+\left(\theta_{2}-\theta_{1}\right) e^{k T_{1}}+\left(\theta_{3}-\theta_{2}\right) e^{k T_{2}}+\cdots+\left(K_{j}-\theta_{i}\right) e^{k T}
$$

It can be easily verified that starting from strikes $K_{j}^{\prime}$ as the initial condition at time $T_{0}=0$, we indeed arrive at the desired strikes at time $T_{i-1}$. Since the number of listed maturities will usually be small (currently around six maturities) and not dependent on the size of the $T$-grid, the additional computational cost will be linear (in the size of the $T$-grid).

As an alternative approach to obtain the initial condition, which is also fast and works well in practice, is to simply perform a convexity-preserving interpolation of the discrete call prices $C\left(K_{j}, T_{i-1}\right)$ along strikes and for the fixed maturity $T_{i-1}$. For example, one can apply the convexity preserving spline interpolation along strikes as described in Fengler (2009).

Finally, we remark an implementation detail regarding the functions $v_{i}(\cdot)$, to be determined for each listed maturity $T_{i}$. In Andreasen and Huge (2011), the authors choose $v_{i}(\cdot)$ as a step-function, with the number of steps equal to the number of market quotes available for maturity $T_{i}$; this ensures that we have enough 'degrees of freedom' to match the given quotes. Alternatively, we found that enough flexibility can be obtained by using simpler parametric forms for $v_{i}(\cdot)$. In our implementation, we have chosen

$$
v_{i}(K)=c_{1}+c_{2} \cdot N\left(\frac{K / V_{0}-c_{3}}{c_{4}}\right)
$$

where $N(\cdot)$ is the standard normal cdf. This has the advantage that it yields a smooth and bounded $v_{i}(K) \in\left[c_{1}, c_{1}+c_{2}\right]$. To find the function $v_{i}(K)$ we now only have to fit the parameter vector $\left(c_{1}, c_{2}, c_{3}, c_{4}\right)$ for each maturity $T_{i}$. Of course, if additional fitting flexibility is required, a less parsimonious specification for $v_{i}(K)$ would need to be used.

\subsubsection{Method II: Indirect method using rolling VIX futures}

The main idea behind the next method will be to obtain the local volatility-of-volatility surface of the VIX spot from that of the VIX futures. Specifically, over each interval $\left[T_{i-1}, T_{i}\right]$, we will compute the local volatility-of-volatility of $F_{t}^{T_{i}}$ (the VIX futures of maturity $\left.T_{i}\right)$ and then obtain $\lambda(K, T)$ from it; accordingly, we will term this method the rolling-VIX futures method. On the interval $\left[T_{i-1}, T_{i}\right]$ the dynamics of $V_{t}$ are:

$$
d V_{t}=k\left(\theta_{i}-V_{t}\right) d t+V_{t} \cdot \lambda\left(V_{t}, t\right) d W_{t}
$$


from where we obtain, for $t \in\left[T_{i-1}, T_{i}\right]$, the VIX futures $F_{t}^{T_{i}}$ as

$$
F_{t}^{T_{i}}=\theta_{i}+\left(V_{t}-\theta_{i}\right) e^{-k\left(T_{i}-t\right)}
$$

with dynamics

$$
d F_{t}^{T_{i}}=e^{-k\left(T_{i}-t\right)} \cdot V_{t} \cdot \lambda\left(V_{t}, t\right) d W_{t}
$$

Now, we can rewrite (23) equivalently as

$$
V_{t}=\theta_{i}+\left(F_{t}^{T_{i}}-\theta_{i}\right) e^{k\left(T_{i}-t\right)}
$$

which, combined with (24), leads to the following VIX futures dynamics:

$$
\begin{aligned}
d F_{t}^{T_{i}} & =e^{-k\left(T_{i}-t\right)}\left(\theta_{i}+\left(F_{t}^{T_{i}}-\theta_{i}\right) e^{k\left(T_{i}-t\right)}\right) \lambda\left(\theta_{i}+\left(F_{t}^{T_{i}}-\theta_{i}\right) e^{k\left(T_{i}-t\right)}, t\right) d W_{t} \\
& \triangleq F_{t}^{T_{i}} \cdot \omega_{i}\left(F_{t}^{T_{i}}, t\right) d W_{t}
\end{aligned}
$$

where we have defined as $\omega_{i}\left(F_{t}^{T_{i}}, t\right)$ the local volatility-of-volatility function of the VIX futures $F_{t}^{T_{i}}$ on the interval $\left[T_{i-1}, T_{i}\right]$. We now consider, for $T \in\left[T_{i-1}, T_{i}\right]$ :

$$
C_{i}(K, T) \triangleq E\left(F_{T}^{T_{i}}-K\right)_{+}
$$

the (undiscounted) call option on $F_{t}^{T_{i}}$ with strike $K$ and maturity $T$. Since $F_{T_{i}}^{T_{i}}=$ $V_{T_{i}}$, VIX options of maturity $T_{i}$ can be viewed as options on the VIX futures $F_{t}^{T_{i}}$. Specifically, using our notation, we have $C_{i}\left(K, T_{i}\right)=e^{r T_{i}} \cdot C\left(K, T_{i}\right)$. For options of maturity $T_{i-1}$, the relation is

$$
\begin{aligned}
C_{i}\left(K, T_{i-1}\right) & =E\left(F_{T_{i-1}}^{T_{i}}-K\right)_{+} \\
& =E\left(\theta_{i}+\left(V_{T_{i-1}}-\theta_{i}\right) e^{-k\left(T_{i}-T_{i-1}\right)}-K\right)_{+} \\
& =e^{-k\left(T_{i}-T_{i-1}\right)} \cdot E\left(V_{T_{i-1}}-\left(\theta_{i}+\left(K-\theta_{i}\right) e^{k\left(T_{i}-T_{i-1}\right)}\right)\right)_{+} \\
& =e^{-k\left(T_{i}-T_{i-1}\right)} \cdot e^{r T_{i-1}} \cdot C\left(\theta_{i}+\left(K-\theta_{i}\right) e^{k\left(T_{i}-T_{i-1}\right)}, T_{i-1}\right) .
\end{aligned}
$$

Since options on the VIX futures must satisfy the standard no-arbitrage conditions (16), we can apply (15) to generate $C_{i}(K, T)$ for $T \in\left(T_{i-1}, T_{i}\right]$, starting from the initial condition (26); note that, in order to obtain the initial condition, a procedure similar to that presented in Method I will be necessary. We can then recover the local 
volatility of volatility function $\omega_{i}(K, T)$ of $F_{t}^{T_{i}}$ from Dupire (1994) formula

$$
\omega_{i}^{2}(K, T)=\frac{\frac{\partial}{\partial T} C_{i}(K, T)}{\frac{1}{2} \cdot K^{2} \cdot \frac{\partial^{2}}{\partial K^{2}} C_{i}(K, T)} .
$$

Finally, from the definition of $\omega_{i}(K, T)$ in (25), we can solve for $\lambda(K, T)$ as

$$
\lambda(K, T)=\frac{\theta_{i}+\left(K-\theta_{i}\right) e^{-k\left(T_{i}-T\right)}}{K} \cdot e^{k\left(T_{i}-T\right)} \cdot \omega_{i}\left(\theta_{i}+\left(K-\theta_{i}\right) e^{-k\left(T_{i}-T\right)}, T\right)
$$

which allows us to obtain the local volatility of volatility function $\lambda(K, T)$ by interpolating $\omega_{i}(K, T)$.

After we have obtained $\omega_{i}(K, T)$ from (27) on a discrete grid, it is important to note that there is a certain degree of arbitrariness in performing the local volatility of volatility interpolation (28): for example we can choose a simple linear interpolation, or a smooth spline interpolation etc., without facing any arbitrage constraints. From an implementation standpoint, we found that Method II will be faster than Method I, but in terms of accuracy some precision can be lost in step (28).

Finally, before concluding this section, we address an idea which may appear a natural variation on our Method II. Instead of rolling the VIX futures for each interval $\left[T_{i-1}, T_{i}\right]$, we could work only with the terminal VIX futures $F_{t}^{T_{N}}$, as follows: (a) translate all market quotes for VIX options to options on the terminal futures, (b) fit the local volatility of volatility $\omega_{N}(K, T)$ and, finally, (c) obtain $\lambda(K, T)$ by interpolating $\omega_{N}(K, T)$ similar to (28). The practical implementation runs into a domain 'shrinkage' problem, due to mean-reversion, as illustrated below.

For clarity, let us assume $\theta(t)=\theta$ (i.e. a constant) and suppose for maturity $T_{1}$ we can observe VIX options quotes at several strikes which lie in the interval $\left[K_{L}, K_{H}\right]$. In terms of the terminal futures, that implies we can observe quotes on the strike interval:

$$
\left[\theta+\left(K_{L}-\theta\right) e^{-k\left(T_{N}-T_{1}\right)}, \theta+\left(K_{H}-\theta\right) e^{-k\left(T_{N}-T_{1}\right)}\right]
$$

Note that the size of this interval, given by $\left(K_{H}-K_{L}\right) e^{-k\left(T_{N}-T_{1}\right)}$, decreases exponentially with the inter-maturity distance $T_{N}-T_{1}$. Now, let us consider a very reasonable numerical example where $k=8, \theta=30 \%,\left[K_{L}, K_{H}\right]=[10 \%, 50 \%]$ and $T_{N}-T_{1}=0.5$ years. The strikes where we can observe market quotes for options on $F_{T_{1}}^{T_{N}}$ will all lie in the tiny interval $[19.63 \%, 20.37 \%]^{3}$ making it numerically difficult to calibrate the local volatility of volatility $\omega_{N}(K, T)$, on the interval $\left[T_{0}, T_{1}\right]$. Most importantly, the interpolation to obtain $\lambda(K, T)$ becomes even more problematic.

3 Note that the shrinkage problem is not alleviated by working in log-strikes:

$$
\lim _{\Delta K \rightarrow 0} \log \left(\frac{\theta+\left(K_{2}-\theta\right) e^{-k\left(T_{N}-T_{1}\right)}}{\theta+\left(K_{1}-\theta\right) e^{-k\left(T_{N}-T_{1}\right)}}\right) / \log \left(\frac{K_{2}}{K_{1}}\right)=e^{-k\left(T_{N}-T_{1}\right)} \cdot \frac{K_{1}}{\theta+\left(K_{1}-\theta\right) e^{-k\left(T_{N}-T_{1}\right)}} .
$$




\section{Implementation and calibration to market data}

In this section, we illustrate the calibration of the local volatility of volatility model to actual market data; the results presented here are based on Method I, as described in the previous section. Recall that our goal is to obtain a global fit to the VIX market, which consists of the following observables: the VIX spot, the term structure of VIX futures and bid/offer prices for VIX call and put options, for all listed expiries. The relevant market quotes are accessible through CBOE's website.

The data set considered below corresponds to CBOE's market close on Jul-052011 and Sep-06-2012; we consider two different dates to allow for better comparison between input data and calibration results. On Jul-05-2011 the VIX spot closed at 16.06 and the following six maturities were listed: Jul-20-2011, Aug-17-2011, Sep21-2011, Oct-19-2011, Nov-16-2011 and Dec-21-2011; on Sep-06-2012 the VIX spot closed at 15.60 and the following six maturities were listed: Sep-19-2012, Oct-172012, Nov-21-2012, Dec-19-2012, Jan-16-2013 and Feb-13-2013. For each maturity, we have a corresponding futures quote as well as several Call and Put options quotes. We have used the VIX futures as an approximate cutoff line between Puts and Calls, as follows: for strikes less than or equal to the VIX futures, we selected the Put options and for strikes larger than the VIX futures, we selected the Call options; all options which showed a non-stale bid and offer, as of the close on Jul-05-2011 and Sep-06-2012, have been considered. The entire data set can be found in "Appendix II'.

The calibration procedure uses mid-market prices, computed as a simple arithmetic average of the bid and offer prices. Also, we converted put prices into call prices using the put-call parity:

$$
C\left(K, T_{i}\right)=e^{-r T_{i}} \cdot\left(F_{0}^{T_{i}}-K\right)+P\left(K, T_{i}\right)
$$

where $F_{0}^{T_{i}}$ is the VIX futures quote for expiry $T_{i}$. In Figs. 3 and 4 below, the black diamonds represent the market VIX option prices, expressed as implied volatilities of volatility, across different strikes and maturities. For Jul-05-2011 we have a total of 77 options quotes, with a range of 10-14 quotes for each maturity; for Sep-06-2012 we have a total of 152 options quotes, with a range of 17-29 quotes for each maturity. We particularly note the steep upward sloping volatilityof-volatility smile, which is an important distinguishing feature of volatility markets.

We first fit the piecewise constant reversion level function $\theta(t)$ to the term structure of VIX futures. For a reversion speed $k=8$, we obtain, by using formula (3) for the six listed maturities the following $\theta_{i}, i \in\{1,2, \ldots, 6\}$ : $\{19.24,19.46,21.65,22.18,22.31,22.07\}$ on Jul-05-2011 and $\{17.62,21.44,22.38$, $23.54,26.53,26.88\}$ on Sep-06-2012. The resulting VIX futures curves are shown in Fig. 2; by construction, we have a perfect fit to the VIX futures term structure.

In order to compute the local volatility of volatility surface $\lambda(K, T)$, we must first perform an arbitrage free interpolation and extrapolation of the available market call prices. For each interval $\left[T_{i-1}, T_{i}\right]$ with $i \in\{1,2, \ldots, 6\}$, we generate the call prices 

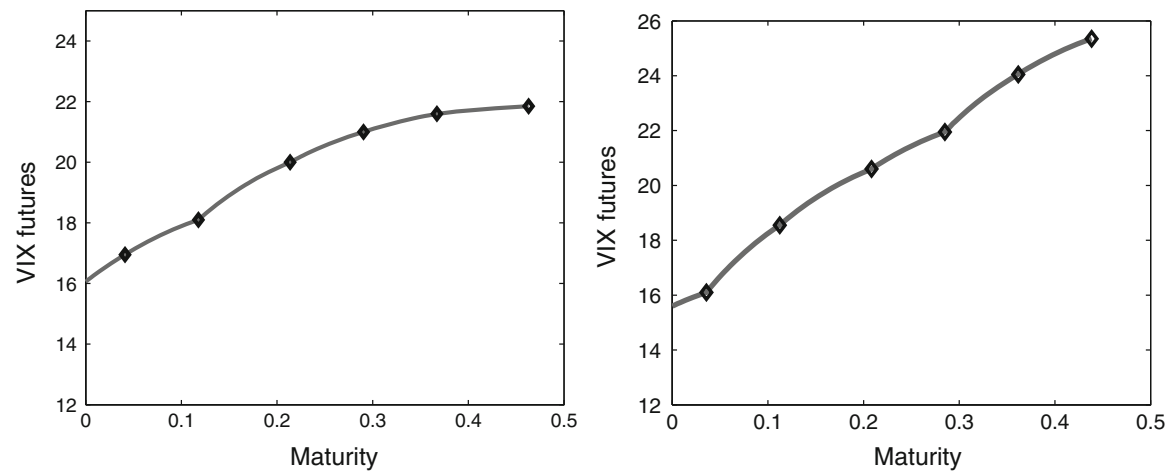

Fig. 2 VIX futures term structure on Jul-05-2011 (left) and Sep-06-2012 (right). Diamond: Market quotes, Solid gray: Model VIX futures curve

using Proposition 3.1 implemented using the algorithm of Andreasen and Huge (2011), properly adjusted as discussed in the previous section. For the functions $v_{i}$, we use our proposed parametric form in (22); this requires to fit only four parameters per maturity and results in a smooth and bounded $v_{i}$.

Figures 3 and 4 show, for each listed maturity $T_{i}$, the implied volatility of volatility generated by the model against the available market quotes. We remark that, despite the very parsimonious specification for the functions $v_{i}$, they offer enough flexibility to obtain satisfactory fits, across strikes and maturities; e.g. the average error across the 77 quotes on Jul-05-2011 has been less than 0.90\%, which compares well to the typical size of the implied volatilities of volatility (normally ranging between $[50 \%, 150 \%])$.

Having generated the arbitrage-free surface of option prices, the final step is the computation of the local volatility of volatility using formula (9). From a numerical standpoint, it is preferable to use this equation rather than the simpler version in (4); especially for extreme strikes, differentiating the implied volatility of volatility gives more stable results (than directly differentiating the absolute call prices).

The resulting local volatility of volatility surfaces are shown in Figs. 5 and 6, for a wide range of VIX values, from 5 to 150 . In agreement with intuition, we note that the local volatility of the VIX retains a similar shape to the implied volatility of volatility curves of Figs. 3 and 4. In particular, the local volatility of the VIX becomes larger when the VIX rises. During periods of market stress, when the VIX rises, the volatility of the VIX also rises substantially.

Finally, we note that in a Matlab implementation, the entire calibration to 6 VIX futures quotes and 77 VIX options quotes from Jul-05-2011 took approximately $45 \mathrm{~s}$ on a laptop equipped with a single Intel Core i5 processor with a processor speed of $2.4 \mathrm{GHz}$; the second data set, containing 152 VIX options quotes from Sep-06-2012, took approximately $65 \mathrm{~s}$.

We have now calibrated the VIX dynamics in (1) to fit the available VIX market quotes; the model can be used to price non-listed VIX products, including exotic derivatives such as VIX barrier options or VIX digital options. 

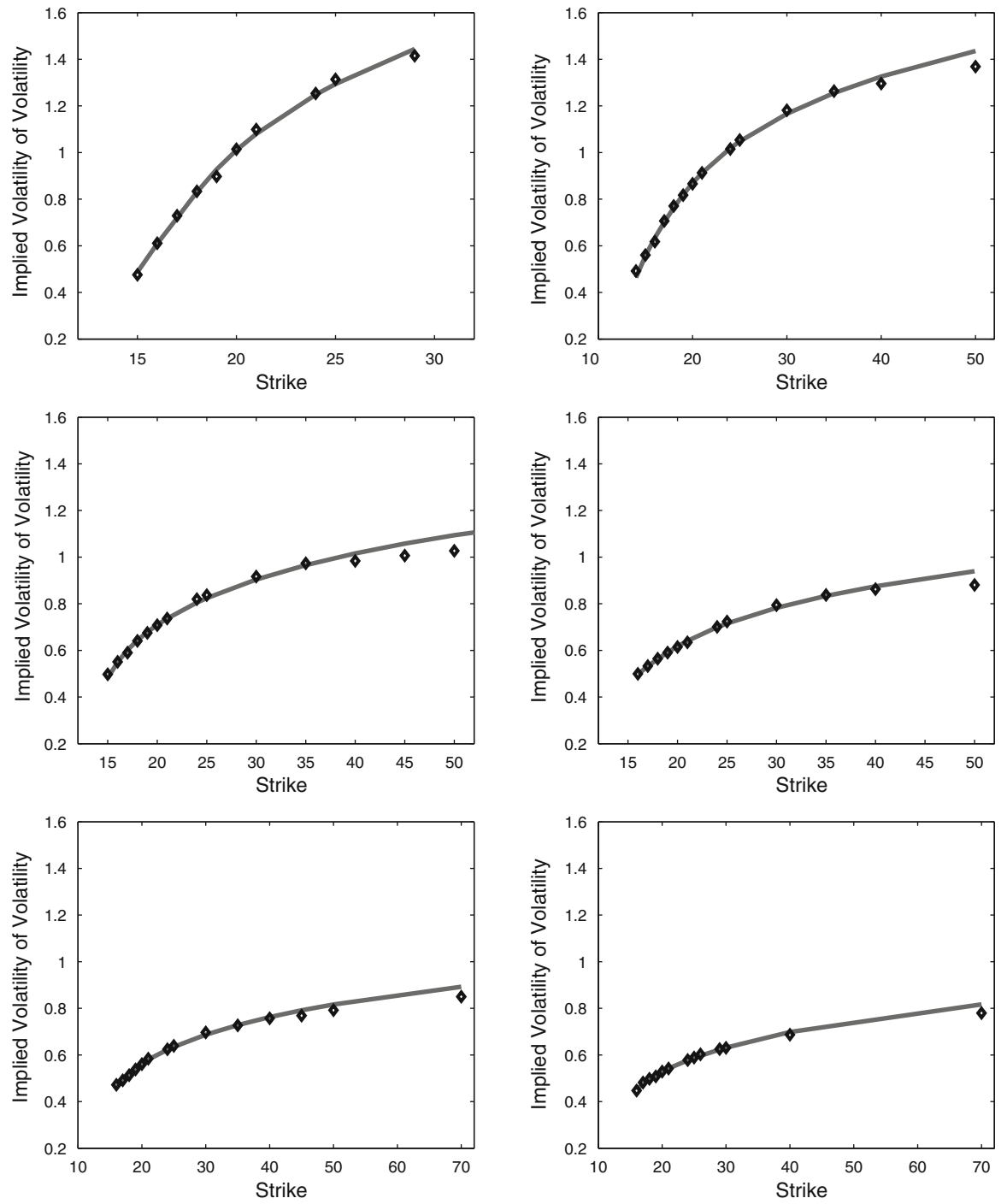

Fig. 3 Implied volatilities of volatility on Jul-05-2011. Diamond: Market quotes, Solid gray: Model generated implied volatility of volatility curves. Maturities, in order from left to right: Jul-20-2011, Aug-17-2011, Sep-21-2011, Oct-19-2011, Nov-16-2011 and Dec-21-2011

\section{Conclusions and further research}

The market in exchange-listed VIX futures and options is becoming a large and mature market, with trading volumes following an exponential growth trend in recent years. Recognizing this development and maturity of the market, we propose to model directly the VIX index itself in a mean reverting local volatility of volatility model; this approach is in contrast with previous literature including traditional stochastic volatil- 

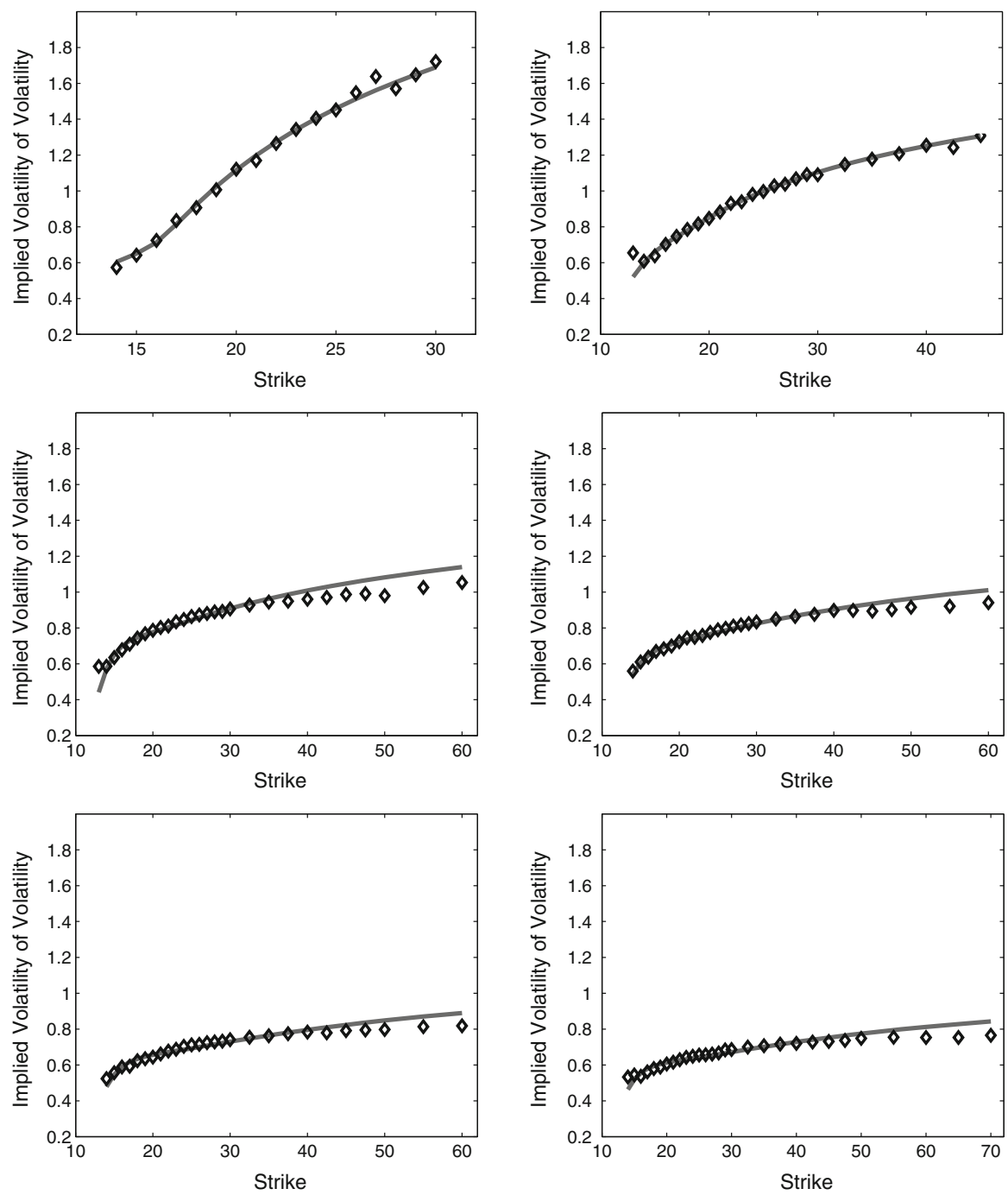

Fig. 4 Implied volatilities of volatility on Sep-06-2012. Diamond: Market quotes, Solid gray: Model generated implied volatility of volatility curves. Maturities, in order from left to right: Sep-19-2012, Oct17-2012, Nov-21-2012, Dec-19-2012, Jan-16-2013 and Feb-13-2013

ity modeling, which focuses on the 'instantaneous' variance. We make the assumption that the VIX market is liquid enough, so that arbitrary VIX derivatives can be priced and hedged relative to the listed (vanilla) VIX instruments.

Assuming linear mean reversion for the VIX index, we first obtain the local volatility of volatility function in terms of the VIX options surface. Then we derive the relevant no arbitrage conditions for VIX options and obtain an algorithm for interpolating and extrapolating the available VIX options quotes to an entire arbitrage free surface. For the latter task, we adapt the ideas in Carr (2008) and Andreasen and Huge 


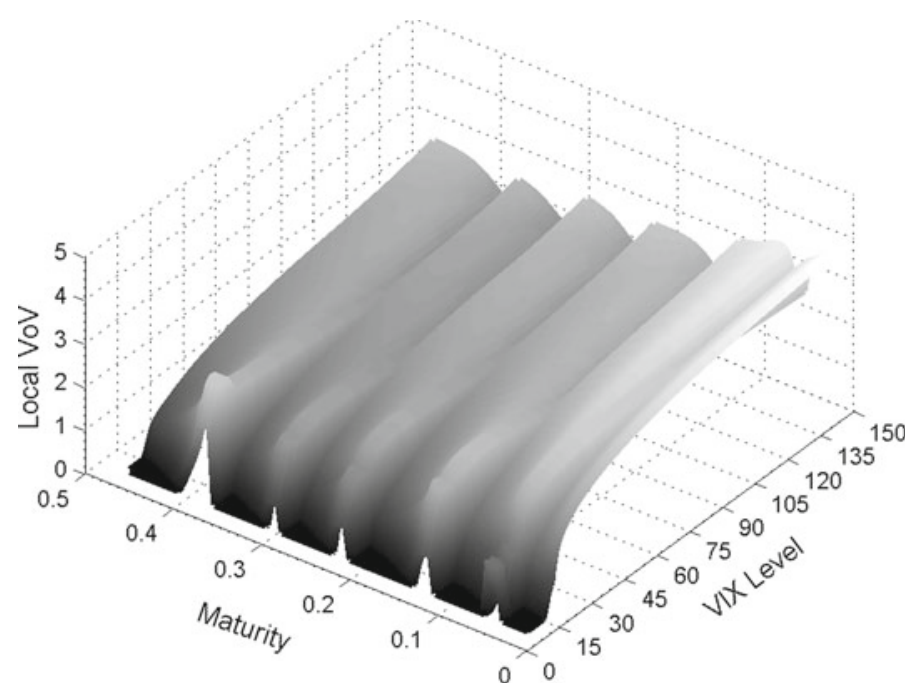

Fig. 5 The local volatility of volatility function $\lambda(V, t)$ from (1) calibrated to Jul-05-2011 VIX market data

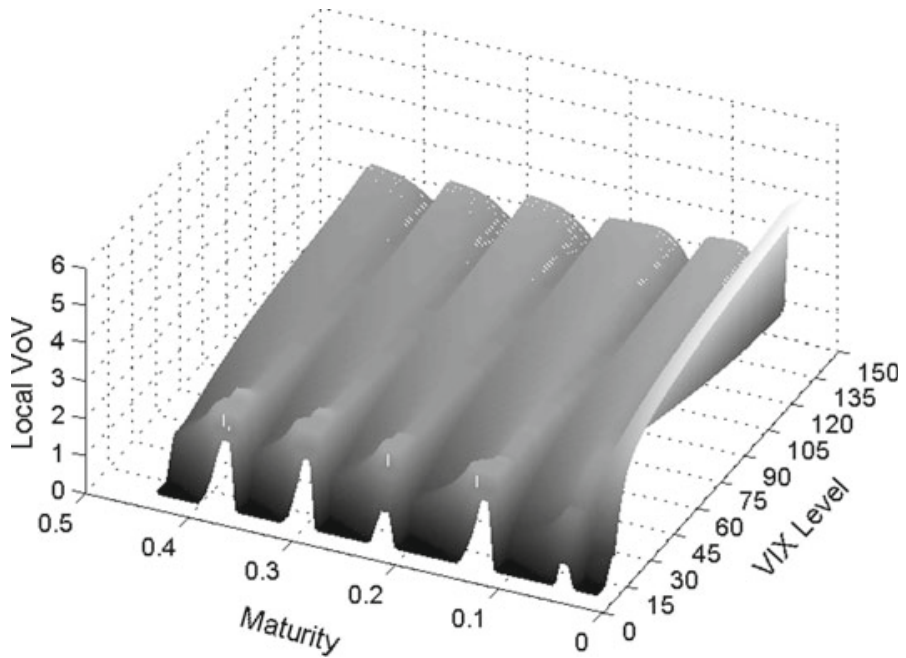

Fig. 6 The local volatility of volatility function $\lambda(V, t)$ from (1) calibrated to Sep-06-2012 VIX market data

(2011) to a mean reverting process. Finally, we analyze the results of the numerical implementation, using CBOE market data.

A further extension of our approach would be to allow for more general non-linear mean reversion in the VIX dynamics-for example quadratic mean reversion as in Carr and Sun (2007) — a topic which we shall leave for future research. Another important avenue for further research is an empirical investigation carried out over a sufficiently long period of time to look at the stability of the calibration, the out-of-sample fitting 
performance as well as the hedging performance in this new modeling framework. All of these topics promise to be of increasing relevance in the future as trading in VIX, as well as other similar volatility indices, continues to develop.

\section{Appendix I}

Proof of Proposition 2.1 Denote $\rho(V, T)$ the density of $V_{T}$. We have

$$
C(K, T)=e^{-r T} \int_{K}^{\infty}(V-K) \cdot \rho(V, T) d V
$$

from where, differentiating with respect to strike $K$ twice

$$
\begin{aligned}
\frac{\partial C}{\partial K}(K, T) & =-e^{-r T} \int_{K}^{\infty} \rho(V, T) d V \\
\frac{\partial^{2} C}{\partial K^{2}}(K, T) & =e^{-r T} \rho(K, T) .
\end{aligned}
$$

Recall Kolmogorov's forward equation for $\rho(V, T)$, see e.g. Jeanblanc et al. (2009):

$$
\frac{\partial \rho}{\partial T}(V, T)=-\frac{\partial}{\partial V}[k(\theta(T)-V) \cdot \rho(V, T)]+\frac{1}{2} \frac{\partial^{2}}{\partial V^{2}}\left[V^{2} \cdot \lambda(V, T)^{2} \cdot \rho(V, T)\right]
$$

Differentiating both sides of (29) with respect to maturity $T$ and using (30)

$$
\begin{aligned}
\frac{\partial C}{\partial T}=- & r C+e^{-r T}\left[-\int_{K}^{\infty}(V-K) \cdot \frac{\partial}{\partial V}[k(\theta(T)-V) \cdot \rho(V, T)] d V\right. \\
& \left.+\frac{1}{2} \int_{K}^{\infty}(V-K) \cdot \frac{\partial^{2}}{\partial V^{2}}\left[V^{2} \cdot \lambda(V, T)^{2} \cdot \rho(V, T)\right] d V\right]
\end{aligned}
$$

Denoting the two terms in the parenthesis by $A$, respectively $B$, we proceed to compute each in turn, using integration by parts. The first term becomes

$$
\begin{aligned}
A & =-\left.(V-K) \cdot[k(\theta(T)-V) \cdot \rho(V, T)]\right|_{K} ^{\infty}+\int_{K}^{\infty} k(\theta(T)-V) \cdot \rho(V, T) d V \\
& =\int_{K}^{\infty} k(\theta(T)-K-(V-K)) \cdot \rho(V, T) d V
\end{aligned}
$$




$$
\begin{aligned}
& =k(\theta(T)-K) \cdot \int_{K}^{\infty} \rho(V, T) d V-k \int_{K}^{\infty}(V-K) \cdot \rho(V, T) d V \\
& =e^{r T}\left[-k(\theta(T)-K) \cdot \frac{\partial C}{\partial K}(K, T)-k \cdot C(K, T)\right]
\end{aligned}
$$

where we have assumed that the boundary term, on the first line, vanishes at $K=+\infty$. The second term becomes

$$
\begin{aligned}
B= & \left.\frac{1}{2}(V-K) \cdot \frac{\partial}{\partial V}\left[V^{2} \cdot \lambda(V, T)^{2} \cdot \rho(V, T)\right]\right|_{K} ^{\infty} \\
& -\frac{1}{2} \int_{K}^{\infty} \frac{\partial}{\partial V}\left[V^{2} \cdot \lambda(V, T)^{2} \cdot \rho(V, T)\right] d V=-\left.\frac{1}{2} V^{2} \cdot \lambda(V, T)^{2} \cdot \rho(V, T)\right|_{K} ^{\infty} \\
& =\frac{1}{2} K^{2} \cdot \lambda(K, T)^{2} \cdot \frac{\partial^{2} C}{\partial K^{2}}(K, T) .
\end{aligned}
$$

where we have assumed that the boundary terms vanish at $K=+\infty$. Using the expressions for $A$ and $B$ in (31), we obtain

$$
\frac{\partial C}{\partial T}=-r C-k(\theta(T)-K) \cdot \frac{\partial C}{\partial K}-k \cdot C+\frac{1}{2} K^{2} \cdot \lambda(K, T)^{2} \cdot \frac{\partial^{2} C}{\partial K^{2}}
$$

which, upon rearrangement, leads to the statement in Proposition 2.1.

Proof of Proposition 2.2 We will find it convenient to work with the undiscounted Black function. Also, noting that, for $T \in\left[T_{i-1}, T_{i}\right]$, the futures price $F_{0}^{T}$ can be written as

$$
F_{0}^{T}=\theta_{i}+e^{-k\left(T-T_{i-1}\right)}\left(F_{0}^{T_{i-1}}-\theta_{i}\right)
$$

the definition of the Black implied volatility surface becomes

$$
e^{r T} \widetilde{C}(x, T) \triangleq C^{B}\left(\theta_{i}+e^{-k\left(T-T_{i-1}\right)}\left(F_{0}^{T_{i-1}}-\theta_{i}\right), V_{0} e^{x}, T, \sigma(x, T)\right) .
$$

For easier reference, we recall below the Black greeks which will be needed in our subsequent calculations:

$$
\begin{array}{llrl}
\frac{\partial C^{B}}{\partial F} & =N\left(d_{1}\right) & & \frac{\partial C^{B}}{\partial K}=-N\left(d_{2}\right) \frac{\partial C^{B}}{\partial T}=\frac{F \sigma n\left(d_{1}\right)}{2 \sqrt{T}} \\
\frac{\partial C^{B}}{\partial \sigma}=F \sqrt{T} n\left(d_{1}\right) & \frac{\partial^{2} C^{B}}{\partial K^{2}}=\frac{F n\left(d_{1}\right)}{\sigma \sqrt{T} K^{2}} \frac{\partial^{2} C^{B}}{\partial \sigma \partial K}=\frac{F d_{1} n\left(d_{1}\right)}{\sigma K} \\
\frac{\partial^{2} C^{B}}{\partial \sigma^{2}}=F \sqrt{T} n\left(d_{1}\right) \frac{d_{1} d_{2}}{\sigma} &
\end{array}
$$

From (32)

$$
e^{r T} \frac{\partial \widetilde{C}}{\partial T}=-r e^{r T} \widetilde{C}+\frac{\partial C^{B}}{\partial F} \cdot \frac{\partial F_{0}^{T}}{\partial T}+\frac{\partial C^{B}}{\partial T}+\frac{\partial C^{B}}{\partial \sigma} \cdot \frac{\partial}{\partial T} \sigma(x, T)
$$




$$
\begin{aligned}
= & -r e^{r T} \widetilde{C}-k e^{-k\left(T-T_{i-1}\right)}\left(F_{0}^{T_{i-1}}-\theta_{i}\right) \cdot N\left(d_{1}\right)+\frac{F_{0}^{T} \sigma(x, T) n\left(d_{1}\right)}{2 \sqrt{T}} \\
& +F_{0}^{T} \sqrt{T} n\left(d_{1}\right) \frac{\partial}{\partial T} \sigma(x, T) . \\
e^{r T} \frac{\partial \widetilde{C}}{\partial x}= & \frac{\partial C^{B}}{\partial K} \cdot V_{0} e^{x}+\frac{\partial C^{B}}{\partial \sigma} \cdot \frac{\partial}{\partial x} \sigma(x, T) \\
= & -N\left(d_{2}\right) \cdot V_{0} e^{x}+F_{0}^{T} \sqrt{T} n\left(d_{1}\right) \frac{\partial}{\partial x} \sigma(x, T) . \\
e^{r T} \frac{\partial^{2} \widetilde{C}}{\partial x^{2}}= & \left(\frac{\partial^{2} C^{B}}{\partial K^{2}} \cdot V_{0} e^{x}+\frac{\partial^{2} C^{B}}{\partial \sigma \partial K} \cdot \frac{\partial}{\partial x} \sigma(x, T)\right) V_{0} e^{x}+\frac{\partial C^{B}}{\partial K} \cdot V_{0} e^{x} \\
& +\left(\frac{\partial^{2} C^{B}}{\partial \sigma \partial K} \cdot V_{0} e^{x}+\frac{\partial^{2} C^{B}}{\partial \sigma^{2}} \cdot \frac{\partial}{\partial x} \sigma(x, T)\right) \frac{\partial}{\partial x} \sigma(x, T)+\frac{\partial C^{B}}{\partial \sigma} \frac{\partial^{2} \sigma}{\partial x^{2}}(x, T) \\
= & \frac{F_{0}^{T} n\left(d_{1}\right)}{\sigma(x, T) \sqrt{T}}+2 \frac{F_{0}^{T} d_{1} n\left(d_{1}\right)}{\sigma(x, T)} \frac{\partial}{\partial x} \sigma(x, T)+F_{0}^{T} \sqrt{T} n\left(d_{1}\right) \frac{d_{1} d_{2}}{\sigma(x, T)}\left(\frac{\partial}{\partial x} \sigma(x, T)\right)^{2} \\
& -N\left(d_{2}\right) V_{0} e^{x}+F_{0}^{T} \sqrt{T} n\left(d_{1}\right) \frac{\partial^{2}}{\partial x^{2}} \sigma(x, T) .
\end{aligned}
$$

Using the expressions in (33), (34) and (35), we obtain for the numerator of Eq. (5)

$$
\begin{aligned}
& \frac{\partial \widetilde{C}}{\partial T}+k\left(\theta_{i}-V_{0} e^{x}\right) \frac{1}{V_{0} e^{x}} \frac{\partial \widetilde{C}}{\partial x}+(r+k) \widetilde{C}=e^{-r T}\left[k \theta_{i}\left(N\left(d_{1}\right)-N\left(d_{2}\right)\right)\right. \\
& \left.+F_{0}^{T} n\left(d_{1}\right)\left(\frac{\sigma(x, T)}{2 \sqrt{T}}+\sqrt{T} \cdot \frac{\partial}{\partial T} \sigma(x, T)+\frac{k\left(\theta_{i}-V_{0} e^{x}\right) \sqrt{T}}{V_{0} e^{x}} \cdot \frac{\partial}{\partial x} \sigma(x, T)\right)\right]
\end{aligned}
$$

and for the denominator of Eq. (5)

$$
\begin{aligned}
& \frac{1}{2} \cdot\left(\frac{\partial^{2} \widetilde{C}}{\partial x^{2}}-\frac{\partial \widetilde{C}}{\partial x}\right)=e^{-r T} \frac{F_{0}^{T}}{2} n\left(d_{1}\right)\left[\frac{1}{\sigma(x, T) \sqrt{T}}+\left(\frac{2 d_{1}}{\sigma(x, T)}-\sqrt{T}\right) \cdot \frac{\partial}{\partial x} \sigma(x, T)\right. \\
& \left.+\frac{\sqrt{T} d_{1} d_{2}}{\sigma(x, T)} \cdot\left(\frac{\partial}{\partial x} \sigma(x, T)\right)^{2}+\sqrt{T} \cdot \frac{\partial^{2}}{\partial x^{2}} \sigma(x, T)\right]
\end{aligned}
$$

Finally, combining (36) and (37), we arrive at the statement in Proposition 2.2.

Proof of Lemma 3.1 The butterfly spread condition (11) is immediate, as the payoff of a butterfly is strictly positive. For the calendar spread condition (10), we consider the VIX futures of maturity $T_{2}$, given by $F_{t}^{T_{2}}=E\left(V_{T_{2}} \mid \mathcal{F}_{t}\right)$, which is a martingale on $\left[0, T_{2}\right]$. By the conditional form of Jensen's inequality

$$
E\left(\left(F_{T_{2}}^{T_{2}}-K\right)_{+} \mid \mathcal{F}_{T_{1}}\right) \geq\left(E\left(F_{T_{2}}^{T_{2}} \mid \mathcal{F}_{T_{1}}\right)-K\right)_{+} \geq\left(F_{T_{1}}^{T_{2}}-K\right)_{+}
$$


which in turn implies

$$
E\left(F_{T_{2}}^{T_{2}}-K\right)_{+} \geq E\left(F_{T_{1}}^{T_{2}}-K\right)_{+}
$$

Using that

$$
\begin{aligned}
& F_{T_{1}}^{T_{2}}=e^{-k\left(T_{2}-T_{1}\right)} \cdot V_{T_{1}}+e^{-k T_{2}} \int_{T_{1}}^{T_{2}} k e^{k t} \theta(t) d t \\
& F_{T_{2}}^{T_{2}}=V_{T_{2}}
\end{aligned}
$$

where the first relation follows by applying Itô to the process $e^{k t} V_{t}$ on $\left[T_{1}, T_{2}\right]$, we obtain

$$
\begin{aligned}
E\left(V_{T_{2}}-K\right)_{+} & \geq E\left(e^{-k\left(T_{2}-T_{1}\right)} \cdot V_{T_{1}}+e^{-k T_{2}} \int_{T_{1}}^{T_{2}} k e^{k t} \theta(t) d t-K\right)_{+} \\
& =e^{-k\left(T_{2}-T_{1}\right)} \cdot E\left[V_{T_{1}}-\left(K e^{k\left(T_{2}-T_{1}\right)}-e^{-k T_{1}} \int_{T_{1}}^{T_{2}} k e^{k t} \theta(t) d t\right)\right]_{+} .
\end{aligned}
$$

Equivalently, this can be written as

$$
e^{r T_{2}} \cdot C\left(K, T_{2}\right) \geq e^{-k\left(T_{2}-T_{1}\right)} \cdot e^{r T_{1}} \cdot C\left(K e^{k\left(T_{2}-T_{1}\right)}-e^{-k T_{1}} \int_{T_{1}}^{T_{2}} k e^{k t} \theta(t) d t, T_{1}\right)
$$

which leads to the statement in Lemma 3.1.

\section{Appendix II: VIX futures and options quotes}

We include below CBOE's VIX futures and options quotes as of the close of trading on Jul-05-2011, when 6 maturities were listed: Jul-20-2011, Aug-17-2011, Sep-21-2011, Oct-19-2011, Nov-16-2011 and Dec-21-2011. The VIX spot closed at 16.06 and the term structure of VIX futures as well as the VIX Put / Call quotes are given in the following tables.

VIX futures term structure

\begin{tabular}{lllllll}
\hline Maturity & $07 / 20 / 2011$ & $08 / 17 / 2011$ & $09 / 21 / 2011$ & $10 / 19 / 2011$ & $11 / 16 / 2011$ & $12 / 21 / 2011$ \\
VIX futures & 16.95 & 18.10 & 20.00 & 21.00 & 21.60 & 21.85 \\
\hline
\end{tabular}


Maturity Jul-20-2011

\begin{tabular}{lllllllllll}
\hline Strike & 15 & 16 & 17 & 18 & 19 & 20 & 21 & 24 & 25 & 29 \\
Bid & 0.05 & 0.4 & 1 & 0.7 & 0.5 & 0.4 & 0.35 & 0.15 & 0.15 & 0.05 \\
Offer & 0.1 & 0.45 & 1.05 & 0.75 & 0.55 & 0.5 & 0.4 & 0.25 & 0.2 & 0.1 \\
Type & $\mathrm{P}$ & $\mathrm{P}$ & $\mathrm{P}$ & $\mathrm{C}$ & $\mathrm{C}$ & $\mathrm{C}$ & $\mathrm{C}$ & $\mathrm{C}$ & $\mathrm{C}$ & $\mathrm{C}$ \\
\hline
\end{tabular}

Maturity Aug-17-2011

\begin{tabular}{|c|c|c|c|c|c|c|c|c|c|c|c|c|c|c|}
\hline Strike & 14 & 15 & 16 & 17 & 18 & 19 & 20 & 21 & 24 & 25 & 30 & 35 & 40 & 50 \\
\hline Bid & 0.05 & 0.25 & 0.6 & 1.15 & 1.8 & 1.6 & 1.4 & 1.2 & 0.8 & 0.75 & 0.45 & 0.25 & 0.15 & 0.05 \\
\hline Offer & 0.1 & 0.3 & 0.65 & 1.25 & 1.9 & 1.7 & 1.45 & 1.3 & 0.9 & 0.8 & 0.5 & 0.35 & 0.2 & 0.1 \\
\hline Type & $\mathrm{P}$ & $\mathrm{P}$ & $\mathrm{P}$ & $\mathrm{P}$ & $\mathrm{P}$ & $\mathrm{C}$ & $\mathrm{C}$ & $\mathrm{C}$ & $\mathrm{C}$ & $\mathrm{C}$ & $\mathrm{C}$ & $\mathrm{C}$ & $\mathrm{C}$ & $\mathrm{C}$ \\
\hline
\end{tabular}

Maturity Sep-21-2011

\begin{tabular}{lllllllllllllll}
\hline Strike & 15 & 16 & 17 & 18 & 19 & 20 & 21 & 24 & 25 & 30 & 35 & 40 & 45 & 50 \\
Bid & 0.15 & 0.45 & 0.8 & 1.35 & 1.9 & 2.55 & 2.2 & 1.6 & 1.45 & 0.85 & 0.55 & 0.3 & 0.2 & 0.1 \\
Offer & 0.25 & 0.5 & 0.9 & 1.4 & 2 & 2.65 & 2.4 & 1.75 & 1.55 & 1 & 0.65 & 0.4 & 0.25 & 0.2 \\
Type & $\mathrm{P}$ & $\mathrm{P}$ & $\mathrm{P}$ & $\mathrm{P}$ & $\mathrm{P}$ & $\mathrm{P}$ & $\mathrm{C}$ & $\mathrm{C}$ & $\mathrm{C}$ & $\mathrm{C}$ & $\mathrm{C}$ & $\mathrm{C}$ & $\mathrm{C}$ & $\mathrm{C}$ \\
\hline
\end{tabular}

Maturity Oct-19-2011

\begin{tabular}{|c|c|c|c|c|c|c|c|c|c|c|c|c|}
\hline Strike & 16 & 17 & 18 & 19 & 20 & 21 & 24 & 25 & 30 & 35 & 40 & 50 \\
\hline Bid & 0.35 & 0.7 & 1.1 & 1.6 & 2.15 & 2.8 & 2 & 1.85 & 1.15 & 0.75 & 0.45 & 0.15 \\
\hline Offer & 0.45 & 0.75 & 1.2 & 1.7 & 2.3 & 2.9 & 2.15 & 1.95 & 1.25 & 0.8 & 0.55 & 0.25 \\
\hline Type & $\mathrm{P}$ & $\mathrm{P}$ & $\mathrm{P}$ & $\mathrm{P}$ & $\mathrm{P}$ & $\mathrm{C}$ & $\mathrm{C}$ & $\mathrm{C}$ & $\mathrm{C}$ & $\mathrm{C}$ & $\mathrm{C}$ & $\mathrm{C}$ \\
\hline
\end{tabular}

Maturity Nov-16-2011

\begin{tabular}{lllllllllllllll}
\hline Strike & 16 & 17 & 18 & 19 & 20 & 21 & 24 & 25 & 30 & 35 & 40 & 45 & 50 & 70 \\
Bid & 0.35 & 0.6 & 1 & 1.45 & 2 & 2.65 & 2.25 & 2.05 & 1.25 & 0.75 & 0.5 & 0.3 & 0.2 & 0.05 \\
Offer & 0.45 & 0.75 & 1.1 & 1.6 & 2.15 & 2.75 & 2.45 & 2.2 & 1.4 & 0.9 & 0.6 & 0.4 & 0.3 & 0.1 \\
Type & $\mathrm{P}$ & $\mathrm{P}$ & $\mathrm{P}$ & $\mathrm{P}$ & $\mathrm{P}$ & $\mathrm{P}$ & $\mathrm{C}$ & $\mathrm{C}$ & $\mathrm{C}$ & $\mathrm{C}$ & $\mathrm{C}$ & $\mathrm{C}$ & $\mathrm{C}$ & $\mathrm{C}$ \\
\hline
\end{tabular}

Maturity Dec-21-2011

\begin{tabular}{llllllllllllll}
\hline Strike & 16 & 17 & 18 & 19 & 20 & 21 & 24 & 25 & 26 & 29 & 30 & 40 & 70 \\
Bid & 0.35 & 0.7 & 1.1 & 1.5 & 2.05 & 2.6 & 2.5 & 2.25 & 2.05 & 1.5 & 1.35 & 0.55 & 0.05 \\
Offer & 0.55 & 0.9 & 1.25 & 1.7 & 2.25 & 2.85 & 2.7 & 2.45 & 2.25 & 1.7 & 1.55 & 0.7 & 0.15 \\
Type & $\mathrm{P}$ & $\mathrm{P}$ & $\mathrm{P}$ & $\mathrm{P}$ & $\mathrm{P}$ & $\mathrm{P}$ & $\mathrm{C}$ & $\mathrm{C}$ & $\mathrm{C}$ & $\mathrm{C}$ & $\mathrm{C}$ & $\mathrm{C}$ & $\mathrm{C}$ \\
\hline
\end{tabular}

We include below CBOE's VIX futures and options quotes as of the close of trading on Sep-06-2012, when 6 maturities were listed: Sep-19-2012, Oct-17-2012, Nov-212012, Dec-19-2012, Jan-16-2013 and Feb-13-2013. The VIX spot closed at 15.60 and the term structure of VIX futures as well as the VIX Put / Call quotes are given in the following tables. 
VIX futures term structure

\begin{tabular}{lllllll}
\hline Maturity & $09 / 19 / 2012$ & $10 / 17 / 2012$ & $11 / 21 / 2012$ & $12 / 19 / 2012$ & $01 / 16 / 2013$ & $02 / 13 / 2012$ \\
VIX futures & 16.10 & 18.55 & 20.60 & 21.00 & 24.05 & 25.35 \\
\hline
\end{tabular}

Maturity Sep-19-2012

\begin{tabular}{llllllllllllllllll}
\hline Strike & 14 & 15 & 16 & 17 & 18 & 19 & 20 & 21 & 22 & 23 & 24 & 25 & 26 & 27 & 28 & 29 & 30 \\
Bid & 0.05 & 0.3 & 0.75 & 0.6 & 0.4 & 0.3 & 0.25 & 0.2 & 0.15 & 0.15 & 0.1 & 0.1 & 0.1 & 0.1 & 0.05 & 0.05 & 0.05 \\
Offer & 0.1 & 0.35 & 0.9 & 0.7 & 0.5 & 0.4 & 0.35 & 0.25 & 0.25 & 0.2 & 0.2 & 0.15 & 0.15 & 0.15 & 0.1 & 0.1 & 0.1 \\
Type & $\mathrm{P}$ & $\mathrm{P}$ & $\mathrm{P}$ & $\mathrm{C}$ & $\mathrm{C}$ & $\mathrm{C}$ & $\mathrm{C}$ & $\mathrm{C}$ & $\mathrm{C}$ & $\mathrm{C}$ & $\mathrm{C}$ & $\mathrm{C}$ & $\mathrm{C}$ & $\mathrm{C}$ & $\mathrm{C}$ & $\mathrm{C}$ & $\mathrm{C}$ \\
\hline
\end{tabular}

Maturity Oct-17-2012

\begin{tabular}{llllllllllllllllll}
\hline Strike & 13 & 14 & 15 & 16 & 17 & 18 & 19 & 20 & 21 & 22 & 23 & 24 & 25 & 26 & 27 & 28 & 29 \\
Bid & 0.05 & 0.1 & 0.25 & 0.6 & 1.05 & 1.6 & 1.75 & 1.5 & 1.25 & 1.1 & 0.9 & 0.8 & 0.65 & 0.6 & 0.5 & 0.45 & 0.4 \\
Offer & 0.1 & 0.15 & 0.35 & 0.7 & 1.15 & 1.7 & 1.9 & 1.55 & 1.35 & 1.2 & 1 & 0.9 & 0.8 & 0.7 & 0.6 & 0.55 & 0.5 \\
Type & $\mathrm{P}$ & $\mathrm{P}$ & $\mathrm{P}$ & $\mathrm{P}$ & $\mathrm{P}$ & $\mathrm{P}$ & $\mathrm{C}$ & $\mathrm{C}$ & $\mathrm{C}$ & $\mathrm{C}$ & $\mathrm{C}$ & $\mathrm{C}$ & $\mathrm{C}$ & $\mathrm{C}$ & $\mathrm{C}$ & $\mathrm{C}$ & \\
Strike & 30 & 32.5 & 35 & 37.5 & 40 & 42.5 & 45 & & & & & & & & & & \\
Bid & 0.35 & 0.25 & 0.15 & 0.1 & 0.1 & 0.05 & 0.05 & & & & & & & & & & \\
Offer & 0.4 & 0.35 & 0.3 & 0.25 & 0.2 & 0.15 & 0.15 & & & & & & & & & & \\
Type & $\mathrm{C}$ & $\mathrm{C}$ & $\mathrm{C}$ & $\mathrm{C}$ & $\mathrm{C}$ & $\mathrm{C}$ & $\mathrm{C}$ & & & & & & & & & & \\
\hline
\end{tabular}

Maturity Nov-21-2012

\begin{tabular}{llllllllllllllllll}
\hline Strike & 13 & 14 & 15 & 16 & 17 & 18 & 19 & 20 & 21 & 22 & 23 & 24 & 25 & 26 & 27 & 28 & 29 \\
Bid & 0.05 & 0.1 & 0.3 & 0.6 & 0.95 & 1.45 & 1.95 & 2.5 & 2.75 & 2.4 & 2.15 & 1.9 & 1.7 & 1.5 & 1.35 & 1.2 & 1.05 \\
Offer & 0.1 & 0.2 & 0.4 & 0.7 & 1.1 & 1.55 & 2.1 & 2.7 & 2.9 & 2.55 & 2.3 & 2.05 & 1.85 & 1.65 & 1.45 & 1.3 & 1.15 \\
Type & $\mathrm{P}$ & $\mathrm{P}$ & $\mathrm{P}$ & $\mathrm{P}$ & $\mathrm{P}$ & $\mathrm{P}$ & $\mathrm{P}$ & $\mathrm{P}$ & $\mathrm{C}$ & $\mathrm{C}$ & $\mathrm{C}$ & $\mathrm{C}$ & $\mathrm{C}$ & $\mathrm{C}$ & $\mathrm{C}$ & $\mathrm{C}$ & $\mathrm{C}$ \\
Strike & 30 & 32.5 & 35 & 37.5 & 40 & 42.5 & 45 & 47.5 & 50 & 55 & 60 & & & & & & \\
Bid & 0.95 & 0.7 & 0.55 & 0.4 & 0.3 & 0.2 & 0.15 & 0.1 & 0.05 & 0.05 & 0.05 & & & & & & \\
Offer & 1.05 & 0.85 & 0.65 & 0.5 & 0.4 & 0.35 & 0.3 & 0.25 & 0.2 & 0.15 & 0.1 & & & & & & \\
Type & $\mathrm{C}$ & $\mathrm{C}$ & $\mathrm{C}$ & $\mathrm{C}$ & $\mathrm{C}$ & $\mathrm{C}$ & $\mathrm{C}$ & $\mathrm{C}$ & $\mathrm{C}$ & $\mathrm{C}$ & $\mathrm{C}$ & & & & & & \\
\hline
\end{tabular}

Maturity Dec-19-2012

\begin{tabular}{llllllllllllllllll}
\hline Strike & 14 & 15 & 16 & 17 & 18 & 19 & 20 & 21 & 22 & 23 & 24 & 25 & 26 & 27 & 28 & 29 & 30 \\
Bid & 0.1 & 0.3 & 0.55 & 0.9 & 1.25 & 1.7 & 2.25 & 2.85 & 3.3 & 3 & 2.75 & 2.5 & 2.25 & 2.05 & 1.85 & 1.7 & 1.55 \\
Offer & 0.2 & 0.4 & 0.65 & 1 & 1.4 & 1.85 & 2.4 & 3 & 3.6 & 3.2 & 2.9 & 2.65 & 2.4 & 2.2 & 2 & 1.8 & 1.65 \\
Type & $\mathrm{P}$ & $\mathrm{P}$ & $\mathrm{P}$ & $\mathrm{P}$ & $\mathrm{P}$ & $\mathrm{P}$ & $\mathrm{P}$ & $\mathrm{P}$ & $\mathrm{C}$ & $\mathrm{C}$ & $\mathrm{C}$ & $\mathrm{C}$ & $\mathrm{C}$ & $\mathrm{C}$ & $\mathrm{C}$ & $\mathrm{C}$ & $\mathrm{C}$ \\
Strike & 32.5 & 35 & 37.5 & 40 & 42.5 & 45 & 47.5 & 50 & 55 & 60 & & & & & & & \\
Bid & 1.2 & 0.95 & 0.75 & 0.65 & 0.5 & 0.4 & 0.3 & 0.25 & 0.15 & 0.1 & & & & & & & \\
Offer & 1.35 & 1.1 & 0.9 & 0.75 & 0.6 & 0.45 & 0.4 & 0.35 & 0.25 & 0.2 & & & & & & & \\
Type & $\mathrm{C}$ & $\mathrm{C}$ & $\mathrm{C}$ & $\mathrm{C}$ & $\mathrm{C}$ & $\mathrm{C}$ & $\mathrm{C}$ & $\mathrm{C}$ & $\mathrm{C}$ & $\mathrm{C}$ & & & & & & & \\
\hline
\end{tabular}


Maturity Jan-16-2013

\begin{tabular}{llllllllllllllllll}
\hline Strike & 14 & 15 & 16 & 17 & 18 & 19 & 20 & 21 & 22 & 23 & 24 & 25 & 26 & 27 & 28 & 29 & 30 \\
Bid & 0.05 & 0.2 & 0.35 & 0.55 & 0.9 & 1.25 & 1.65 & 2.15 & 2.7 & 3.3 & 3.9 & 3.6 & 3.2 & 2.95 & 2.7 & 2.45 & 2.25 \\
Offer & 0.15 & 0.25 & 0.5 & 0.7 & 1.05 & 1.4 & 1.8 & 2.3 & 2.85 & 3.4 & 4.1 & 3.8 & 3.5 & 3.2 & 2.9 & 2.65 & 2.45 \\
Type & $\mathrm{P}$ & $\mathrm{P}$ & $\mathrm{P}$ & $\mathrm{P}$ & $\mathrm{P}$ & $\mathrm{P}$ & $\mathrm{P}$ & $\mathrm{P}$ & $\mathrm{P}$ & $\mathrm{P}$ & $\mathrm{P}$ & $\mathrm{C}$ & $\mathrm{C}$ & $\mathrm{C}$ & $\mathrm{C}$ & $\mathrm{C}$ & $\mathrm{C}$ \\
Strike & 32.5 & 35 & 37.5 & 40 & 42.5 & 45 & 47.5 & 50 & 55 & 60 & & & & & & & \\
Bid & 1.8 & 1.45 & 1.15 & 0.95 & 0.75 & 0.6 & 0.5 & 0.4 & 0.25 & 0.15 & & & & & & & \\
Offer & 2 & 1.6 & 1.35 & 1.1 & 0.85 & 0.75 & 0.6 & 0.5 & 0.4 & 0.3 & & & & & & & \\
Type & $\mathrm{C}$ & $\mathrm{C}$ & $\mathrm{C}$ & $\mathrm{C}$ & $\mathrm{C}$ & $\mathrm{C}$ & $\mathrm{C}$ & $\mathrm{C}$ & $\mathrm{C}$ & $\mathrm{C}$ & & & & & & & \\
\hline
\end{tabular}

\begin{tabular}{llllllllllllllllll}
\hline \multicolumn{1}{l}{ Maturity Feb-13-2013 } \\
\hline Strike & 14 & 15 & 16 & 17 & 18 & 19 & 20 & 21 & 22 & 23 & 24 & 25 & 26 & 27 & 28 & 29 & 30 \\
Bid & 0.05 & 0.15 & 0.3 & 0.5 & 0.75 & 1.05 & 1.45 & 1.85 & 2.35 & 2.9 & 3.4 & 4 & 4 & 3.6 & 3.3 & 3.1 & 2.85 \\
Offer & 0.2 & 0.3 & 0.35 & 0.6 & 0.9 & 1.2 & 1.6 & 2.05 & 2.55 & 3.1 & 3.7 & 4.3 & 4.2 & 3.9 & 3.6 & 3.4 & 3.1 \\
Type & $\mathrm{P}$ & $\mathrm{P}$ & $\mathrm{P}$ & $\mathrm{P}$ & $\mathrm{P}$ & $\mathrm{P}$ & $\mathrm{P}$ & $\mathrm{P}$ & $\mathrm{P}$ & $\mathrm{P}$ & $\mathrm{P}$ & $\mathrm{P}$ & $\mathrm{C}$ & $\mathrm{C}$ & $\mathrm{C}$ & $\mathrm{C}$ & $\mathrm{C}$ \\
Strike & 32.5 & 35 & 37.5 & 40 & 42.5 & 45 & 47.5 & 50 & 55 & 60 & 65 & 70 & & & & & \\
Bid & 2.35 & 1.9 & 1.55 & 1.25 & 1.05 & 0.85 & 0.7 & 0.6 & 0.4 & 0.25 & 0.15 & 0.15 & & & & \\
Offer & 2.55 & 2.1 & 1.75 & 1.45 & 1.2 & 1 & 0.85 & 0.75 & 0.55 & 0.4 & 0.3 & 0.2 & & & & \\
Type & $\mathrm{C}$ & $\mathrm{C}$ & $\mathrm{C}$ & $\mathrm{C}$ & $\mathrm{C}$ & $\mathrm{C}$ & $\mathrm{C}$ & $\mathrm{C}$ & $\mathrm{C}$ & $\mathrm{C}$ & $\mathrm{C}$ & $\mathrm{C}$ & & & & \\
\hline
\end{tabular}

\section{References}

Andersen, L., \& Brotherton-Ratcliffe, R. (1998). The equity option volatility smile: An implicit finitedifference approach. Journal of Computational, Finance, 1(2), 5-37.

Andersen, L., \& Andreasen, J. (2000). Jump diffusion processes: Volatility smile fitting and numerical methods for options pricing. Review of Derivatives Research, 4(3), 231-262.

Andreasen, J., \& Huge, B. (2011). Volatility interpolation. Risk Magazine, pp. 76-79.

Bergomi, L. (2005). Smile dynamics 2. Risk Magazine, pp. 67-73.

Bergomi, L. (2008). Smile dynamics 3. Risk Magazine, pp. 90-96.

Black, F., \& Scholes, M. (1973). The pricing of options and corporate liabilities. Journal of Political Economy, 81, 637-654.

Carr, P., \& Madan, D. (1998). Towards a theory of volatility trading. In R. Jarrow (Ed.), Volatility: New estimation techniques for pricing derivatives (pp. 417-427). London: Risk Books.

Carr, P., \& Sun, J. (2007). A new approach for option pricing under stochastic volatility. Review of Derivatives Research, 10, 87-150.

Carr, P. (2008). Local Variance gamma, working paper. New York: Bloomberg Quant Research.

Cont, R., \& Kokholm, T. (forthcoming, 2011). A consistent pricing model for index options and volatility derivatives. Mathematical Finance.

Derman, E., \& Kani, I. (1994). Riding on a smile. Risk Magazine, 7(2), 32-39.

Dupire, B. (1993). Model art. Risk Magazine, 6(9), 118-124.

Dupire, B. (1994). Pricing with a smile. Risk Magazine, 7(1), 18-20.

Eberlein, E., \& Madan, D. (2009). Sato processes and the valuation of structured products. Quantitative Finance, 9(1), 27-42.

Fengler, M. (2009). Arbitrage-free smoothing of the implied volatility surface. Quantitative Finance, 9(4), 417-428.

Heston, S. (1993). A closed-form solution for options with stochastic volatility with applications to bond and currency options. The Review of Financial Studies, 6, 327-343.

Jeanblanc, M., Yor, M., \& Chesney, M. (2009). Mathematical methods for financial markets. London: Springer Finance.

Lewis, A. (2000). Option valuation under stochastic volatility. Newport Beach, CA: Finance Press.

Neuberger, A. (1994). The log contract. Journal of Portfolio Management, 20, 2. 
Scott, L. (1987). Option pricing when the variance changes randomly: Theory, estimation and an application. Journal of Financial and Quantitative Analysis, 22, 419-438.

Szado, E. (2009). VIX futures and options: A case study of portfolio diversification during the 2008 financial crisis. Journal of Alternative Investments, 12(2), 68-85. 